\title{
The Interval Between Staged Bilateral Total Knee Arthroplasties Does Not Affect Early Complications of the Second Knee or Long-term Function of the First and Second Knees
}

\section{Guangqian Shang}

The Affiliated Hospital of Qingdao University

\section{Shuai Xiang}

The Affiliated Hospital of Qingdao University

Jianjun Guo

The Affiliated Hospital of Qingdao University

Feng Ji

The Affiliated Hospital of Qingdao University

\section{Shuai Yang}

The Affiliated Hospital of Qingdao University

Kun Liu

The Affiliated Hospital of Qingdao University

Hao Xu (D18661806627@163.com )

The Affiliated Hospital of Qingdao University

\section{Research article}

Keywords: Staged, Bilateral total knee arthroplasty, Complications, Functional outcome

Posted Date: November 15th, 2021

DOI: https://doi.org/10.21203/rs.3.rs-1069755/v1

License: (a) (i) This work is licensed under a Creative Commons Attribution 4.0 International License. Read Full License 


\section{Abstract}

Background: This study explored the optimal time interval between staged bilateral total knee arthroplasty (BTKA) to minimise early complications of the second TKA and maximise long-term function of the first and second knees.

Methods: We retrospectively reviewed 266 patients who underwent staged BTKA between 2010 and 2015 . Groups 1-4 had time intervals between BTKAs of 1-6,6-12,12-18, and 18-24 months, respectively. Demographics, postoperative complications within 90 days of the second TKA, Knee Society Score (KSS), and Western Ontario and McMaster Universities Arthritis Index (WOMAC) score were compared among the groups.

Results: In total, 54, 96, 75, and 41 patients were assigned to groups 1-4, respectively. Although group 1 had the highest overall complication rate $(11.11 \%)$, there was no significant difference in the complication rate among the four groups. Also, no significant differences were found among the four groups in functional and patient-reported outcomes, in either the first or second knee at 5 years postoperatively, including KSS-knee, KSS-function, WOMAC-pain, WOMAC-stiffness, and WOMACphysical function. The interval between BTKA did not influence complications or the function of the second knee. The TKA type (posterior-stabilised vs. medial-pivot) and age did not correlate significantly with any scores.

Conclusions: There was no group difference in early complications of the second TKA, and postoperative function was equivalent between the two knees and did not vary by the interval between surgeries. Our results can help surgeons discuss the timing of the second TKA with patients, which should be based on their own preferences. If patients cannot tolerate severe symptoms in the contralateral knee after the first TKA, the second TKA should be performed as early as possible.

\section{Background}

Total knee arthroplasty (TKA) is an effective treatment for end-stage knee osteoarthritis (OA), relieving pain and improving joint function and patient quality of life. The number of TKA will increase in coming decades, with advances in surgical technology and prostheses and the increasing size of the elderly population. One-third of patients with knee OA have bilateral symptoms and up to $19 \%$ require bilateral TKA (BTKA) due to severe bilateral knee destruction [1, 2].

BTKA can be performed simultaneously under the same anaesthetic or staged over different hospital admissions. There has been continual debate about the best approach in terms of economics, perioperative complications, and postoperative joint function; no consensus has been reached. Although simultaneous BTKA is superior in terms length of stay and costs, a growing body of research recommends staged BTKA as a safe, efficacious treatment with fewer complications and less mortality [3-5]. According to the Canadian Hospital Morbidity Database, the annual number of staged BTKA increased by $28 \%$, while simultaneous BTKA decreased by $8 \%$ [6]. 
Currently, the interval between staged BTKA varies and the timing of the second TKA depends largely on patient preference. A literature review found little evidence regarding whether the interval affects the functional outcomes of both knees [7-9]. While three studies have evaluated the optimal timing of the second TKA to minimise early complications, there are no concrete guidelines [9-11].

Therefore, this study explored the optimal interval between staged BTKA to minimise early complications of the second TKA and maximise long-term functional outcomes of both knees.

\section{Materials And Methods}

This retrospective cohort study was approved by our Institutional Review Board. We reviewed our departmental electronic medical record database of arthroplasty patients and identified those who underwent staged BTKA between 2010 and 2015. This database includes clinical and radiological data for patients who underwent annual postoperative follow-ups. The inclusion criteria were a diagnosis of bilateral Kellgren-Lawrence stage III or IV knee OA with related symptoms before the first TKA, staged bilateral primary TKA performed by the same surgeon (not during the same hospitalisation), and a minimum postoperative follow-up of 5 years for each knee. Exclusion criteria were posttraumatic or inflammatory arthritis, the need for a constrained prosthesis in either knee, simultaneous BTKA, and staged BTKA during the same hospitalisation.

Patients who met the inclusion criteria were divided into groups 1-4 according to the interval between surgeries: $1-6,6-12,12-18$, and 18-24 months, respectively. The timing of the second TKA was entirely up to the patient, and depended on their physical and financial situation (e.g. ability to tolerate additional pain and limitations in activities of daily living).

The demographic information obtained included gender, age, body mass index (BMI), Charlson Comorbidity Index (CCl), and TKA type. The $\mathrm{CCl}$ is calculated by summing the weighted scores for 19 medical conditions to assess underlying comorbidities [12]. The postoperative surgical and medical complications within 90 days of the second TKA used as outcome measures included wound complications, deep vein thrombosis, periprosthetic joint infection (PJI), and urinary, cardiac, pulmonary, and cerebral complications. PJI was identified based on the Musculoskeletal Infection Society definition [13]. Functional and patient-reported outcomes of both knees were assessed preoperatively and 5 years postoperatively using the Knee Society Score (KSS) [14] and Western Ontario and McMaster Universities Arthritis Index (WOMAC) score [15]. The KSS is divided into knee and function scores, both of which range from 0 to 100, with higher scores representing better outcomes. For the WOMAC, a higher score indicates poorer condition. There are 24 questions and three subscales: pain (5 questions), stiffness (2 questions), and physical function (17 questions).

Although the TKA procedures were performed by five experienced arthroplasty surgeons, all patients received the same prosthesis, which was placed in both knees by the same surgeon. Two different total knee systems were used: posterior-stabilised (PS; NexGen; Zimmer, Warsaw, IN, USA) and medial-pivot (MP; Advance, MicroPort, Arlington, TN, USA). All patients underwent general anaesthesia combined with 
peripheral nerve blocks, using a thigh tourniquet inflated to $100 \mathrm{~mm} \mathrm{Hg}$ above the systolic pressure, an anterior midline skin incision, and medial parapatellar or mid-vastus approach. Patellar arthroplasty was not done in any patient, although peripatellar osteophytes were routinely trimmed following denervation. A drainage tube was placed before skin closure and removed 24-48 hours postoperatively.

All patients underwent the same postoperative management, including 48 hours of intravenous cefazolin or clindamycin to prevent infection (started 1 hour before the skin incision), $10 \mathrm{mg}$ oral rivaroxaban once daily for 30 days to prevent venous thrombus embolism (started within 12-24 hours postoperatively), continuous passive motion exercises starting on the first postoperative day, and full weight-bearing and walking with a walking-aid on the second postoperative day.

\section{Statistical analysis}

The statistical analyses were performed using SPSS 23.0 (IBM Corp., Armonk, NY, USA). The normality of the data distribution was determined by the Kolmogorov-Smirnov test. Continuous and categorical variables are expressed as means and standard deviations, and frequencies and percentages, respectively. The paired $t$-test was used to compare continuous variables between the first and second knees. One-way analysis of variance was used to compare continuous variables among groups. The chisquare test was used to compare categorical variables. Correlations were assessed using the Pearson coefficient. $P<0.05$ indicated statistical significance.

\section{Results}

This retrospective cohort study enrolled 266 patients who underwent staged BTKA, 54, 96, 75, and 41 were done with intervals of 1-6 (group 1), 6-12 (group 2), 12-18 (group 3), and 18-24 (group 4) months, respectively. Table 1 summarises the preoperative data. There was no significant group difference in gender, age, BMI, CCl, TKA type, KSS, or WOMAC score (all $p>0.05$ ), except the KSS-knee scire for the second knee $(p=0.002)$. In group 4 , there were significant differences between the first and second knees for all preoperative scores (all $p<0.05)$, and for KSS-function $(p=0.069)$ and WOMACphysical function $(p=0.179)$. 
Table 1

Preoperative data between groups and between the first and second knees

\begin{tabular}{|c|c|c|c|c|c|}
\hline & $\begin{array}{l}\text { Group } 1 \\
(n=54)\end{array}$ & $\begin{array}{l}\text { Group } 2 \\
(n=96)\end{array}$ & $\begin{array}{l}\text { Group } 3 \\
(n=75)\end{array}$ & $\begin{array}{l}\text { Group } 4 \\
(n=41)\end{array}$ & $P$ value \\
\hline \multicolumn{6}{|l|}{ Gender (F/M) } \\
\hline Female $(\mathrm{n}, \%)$ & 47 (87.04) & 80 (83.33) & 64 (85.33) & 33 (80.49) & \multirow[t]{2}{*}{0.831} \\
\hline Male (n, \%) & $7(12.96)$ & 16 (16.67) & 11 (14.67) & $8(19.51)$ & \\
\hline Age (year) & $67.85 \pm 8.31$ & $68.90 \pm 7.80$ & $70.43 \pm 7.25$ & $68.42 \pm 7.68$ & 0.266 \\
\hline \multicolumn{6}{|l|}{ BMI $\left(\mathrm{kg} / \mathrm{m}^{2}\right)$} \\
\hline 1st knee & $26.74 \pm 4.04$ & $26.22 \pm 5.00$ & $26.81 \pm 4.75$ & $27.33 \pm 5.80$ & 0.654 \\
\hline 2nd knee & $27.03 \pm 4.04$ & $26.35 \pm 5.02$ & $26.97 \pm 4.82$ & $27.15 \pm 5.71$ & 0.745 \\
\hline$P$ value & 0.701 & 0.858 & 0.837 & 0.890 & \\
\hline $\mathrm{CCl}$ & $2.57 \pm 1.24$ & $2.33 \pm 1.38$ & $2.52 \pm 1.35$ & $2.29 \pm 1.40$ & 0.600 \\
\hline \multicolumn{6}{|l|}{ The TKA types } \\
\hline $\mathrm{MP}(\mathrm{n}, \%)$ & 32 (59.26) & 62 (64.58) & 40 (53.33) & $24(58.54)$ & \multirow[t]{2}{*}{0.531} \\
\hline PS (n, \%) & $22(40.74)$ & 34 (35.42) & 35 (46.67) & $17(41.46)$ & \\
\hline \multicolumn{6}{|l|}{ KSS-knee } \\
\hline 1st knee & $36.80 \pm 11.22$ & $38.26 \pm 10.74$ & $37.87 \pm 11.49$ & $38.88 \pm 13.08$ & 0.827 \\
\hline 2nd knee & $38.22 \pm 10.55$ & $39.79 \pm 9.80$ & $42.91 \pm 7.79$ & $44.37 \pm 7.30$ & 0.002 \\
\hline$P$ value & 0.498 & 0.303 & 0.002 & 0.022 & \\
\hline \multicolumn{6}{|l|}{ KSS-function } \\
\hline 1st knee & $38.33 \pm 13.21$ & $40.47 \pm 13.67$ & $39.20 \pm 13.15$ & $40.12 \pm 14.25$ & 0.802 \\
\hline 2nd knee & $39.54 \pm 12.41$ & $41.88 \pm 12.74$ & $42.27 \pm 10.50$ & $45.24 \pm 10.66$ & 0.140 \\
\hline$P$ value & 0.627 & 0.462 & 0.117 & 0.069 & \\
\hline \multicolumn{6}{|l|}{ WOMAC-pain } \\
\hline 1st knee & $17.96 \pm 4.47$ & $16.80 \pm 3.82$ & $17.39 \pm 3.82$ & $16.85 \pm 4.29$ & 0.345 \\
\hline 2nd knee & $17.13 \pm 4.46$ & $16.41 \pm 3.71$ & $16.79 \pm 3.51$ & $15.12 \pm 3.47$ & 0.063 \\
\hline$P$ value & 0.334 & 0.467 & 0.318 & 0.048 & \\
\hline
\end{tabular}

BMI: body mass index, TKA: total knee arthroplasty, KSS: Knee Society Score, WOMAC: Western Ontario and McMaster Universities Arthritis Index 


\begin{tabular}{|llllll|}
\hline & $\begin{array}{l}\text { Group 1 } \\
(\mathbf{n = 5 4 )}\end{array}$ & $\begin{array}{l}\text { Group 2 } \\
(\mathbf{n = 9 6 )}\end{array}$ & $\begin{array}{l}\text { Group 3 } \\
(\mathbf{n = 7 5 )}\end{array}$ & $\begin{array}{l}\text { Group 4 } \\
(\mathbf{n = 4 1 )}\end{array}$ & P value \\
\hline WOMAC-stiffness & & & & \\
\hline 1st knee & $6.94 \pm 1.84$ & $7.28 \pm 2.11$ & $7.15 \pm 2.06$ & $6.83 \pm 2.04$ & 0.605 \\
\hline 2nd knee & $6.39 \pm 1.72$ & $6.63 \pm 1.88$ & $6.47 \pm 1.72$ & $5.95 \pm 1.63$ & 0.239 \\
\hline P value & 0.108 & 0.024 & 0.030 & 0.034 & \\
\hline WOMAC-physical function & & & & 0.852 \\
\hline 1st knee & $57.48 \pm 13.64$ & $55.69 \pm 14.25$ & $57.24 \pm 13.87$ & $56.49 \pm 13.61$ & 0.666 \\
\hline 2nd knee & $56.11 \pm 13.32$ & $54.34 \pm 14.18$ & $54.45 \pm 12.56$ & $52.66 \pm 13.01$ & \\
\hline \begin{tabular}{l} 
P value \\
\hline
\end{tabular} & 0.598 & 0.513 & 0.199 & & \\
\hline
\end{tabular}

Table 2 summarises complications within 90 days of the second TKA. Nineteen complications were identified after the second TKA, including six urinary complications (31.58\%), four wound complications (21.05\%), two PJls, cardiac complications, pulmonary complications, and cerebral complications, and one deep vein thrombosis. Although group 1 had the highest overall complication rate, of $11.11 \%$, there was no significant difference in the rate of complications among the four groups $(p=0.582)$. 
Table 2

Complications within 90 days of the second total knee arthroplasty between groups

\begin{tabular}{|llllll|}
\hline complications (n, \%) & $\begin{array}{l}\text { Group 1 } \\
(\mathbf{n}=54)\end{array}$ & $\begin{array}{l}\text { Group 2 } \\
(\mathbf{n}=96)\end{array}$ & $\begin{array}{l}\text { Group 3 } \\
(\mathbf{n}=75)\end{array}$ & $\begin{array}{l}\text { Group 4 } \\
(\mathbf{n}=41)\end{array}$ & P value \\
\hline Wound complications & $1(1.85)$ & $2(2.08)$ & 0 & $1(2.44)$ & 0.652 \\
\hline DVT & $1(1.85)$ & 0 & 0 & 0 & 0.270 \\
\hline PJI & $1(1.85)$ & $1(1.04)$ & 0 & 0 & 0.605 \\
\hline Urinary & $2(3.70)$ & $2(3.13)$ & $2(2.67)$ & 0 & 0.679 \\
\hline Cardiac & 0 & $1(1.04)$ & $1(1.33)$ & 0 & 0.764 \\
\hline Pulmonary & $1(1.85)$ & 0 & 0 & $1(2.44)$ & 0.294 \\
\hline Cerebral & 0 & $1(2.08)$ & $1(1.33)$ & 0 & 0.764 \\
\hline Overall complications & $6(11.11)$ & $7(7.29)$ & $4(5.33)$ & $2(4.88)$ & 0.582 \\
\hline DVT : deep vein thrombosis, PJI: periprosthetic joint infection & \\
\hline
\end{tabular}

The postoperative scores of four patients (two in group 2 and one each in groups 1 and 4) were not determined because PJI occurred in two patients each within and after more than 90 days following the second TKA. All knees in all groups improved from preoperatively to 5 years postoperatively. Table 3 shows the postoperative outcomes of the four groups, and compares the outcomes between the first and second knees. There were no significant group differences in KSS-pain, KSS-function, WOMAC-pain, WOMAC-stiffness, or WOMAC-physical function in either the first or second knees (all $p>0.05$ ). 
Table 3

Postoperative outcomes between interval groups and between the first and second knee

$\begin{array}{lllll}\text { Group 1 } & \text { Group 2 } & \text { Group 3 } & \text { Group 4 } & \text { P value } \\ (n=53) & (n=94) & (n=75) & (n=40) & \end{array}$

KSS-knee score

\begin{tabular}{llllll}
\hline 1st knee & $86.40 \pm 6.53$ & $85.33 \pm 6.63$ & $85.76 \pm 6.37$ & $86.15 \pm 6.14$ & 0.785 \\
\hline 2nd knee & $85.89 \pm 6.50$ & $86.03 \pm 6.15$ & $86.72 \pm 5.60$ & $87.45 \pm 5.53$ & 0.534 \\
\hline P value & 0.688 & 0.452 & 0.329 & 0.323 &
\end{tabular}

KSS-function score

\begin{tabular}{|c|c|c|c|c|c|}
\hline 1st knee & $86.79 \pm 9.36$ & $86.17 \pm 8.56$ & $85.47 \pm 8.74$ & $87.25 \pm 8.16$ & 0.719 \\
\hline 2nd knee & $87.74 \pm 8.24$ & $87.77 \pm 7.92$ & $86.80 \pm 8.57$ & $86.50 \pm 8.02$ & 0.773 \\
\hline$P$ value & 0.583 & 0.186 & 0.347 & 0.680 & \\
\hline \multicolumn{6}{|c|}{ WOMAC-pain } \\
\hline 1st knee & $4.49 \pm 1.40$ & $4.73 \pm 1.46$ & $4.65 \pm 1.38$ & $4.25 \pm 1.72$ & 0.332 \\
\hline 2nd knee & $4.28 \pm 1.47$ & $4.40 \pm 1.50$ & $4.47 \pm 1.47$ & $3.95 \pm 1.54$ & 0.322 \\
\hline$P$ value & 0.458 & 0.128 & 0.425 & 0.413 & \\
\hline \multicolumn{6}{|c|}{ WOMAC-stiffness } \\
\hline 1st knee & $1.91 \pm 1.08$ & $2.27 \pm 1.18$ & $2.11 \pm 1.17$ & $2.35 \pm 1.23$ & 0.214 \\
\hline 2nd knee & $2.11 \pm 0.99$ & $2.21 \pm 1.23$ & $1.96 \pm 1.17$ & $2.25 \pm 1.06$ & 0.459 \\
\hline$P$ value & 0.305 & 0.763 & 0.443 & 0.698 & \\
\hline \multicolumn{6}{|c|}{ WOMAC-physical function } \\
\hline 1st knee & $18.32 \pm 4.89$ & $19.65 \pm 4.24$ & $19.95 \pm 4.56$ & $18.78 \pm 4.67$ & 0.169 \\
\hline 2nd knee & $17.98 \pm 4.69$ & $18.69 \pm 3.98$ & $19.37 \pm 4.15$ & $18.95 \pm 3.86$ & 0.312 \\
\hline$P$ value & 0.716 & 0.112 & 0.422 & 0.855 & \\
\hline
\end{tabular}

KSS: Knee Society Score, WOMAC: Western Ontario and McMaster Universities Arthritis Index

The interval between surgeries did not influence complications $(r=-0.080, p=0.192)$, KSS-knee $(r=0.088$, $p=0.156)$, KSS-function $(r=-0.059, p=0.342)$, WOMAC-pain $(r=-0.035, p=0.574)$, WOMAC-stiffness $(r=$ $-0.012, p=0.845)$, or WOMAC-physical function $(r=-0.092, p=0.139)$ of the second knee postoperatively. The TKA type (MP or PS) did not correlate with postoperative KSS-knee $(r=0.02, p=0.974$ and $r=0.013$, $p=0.828$, respectively) or KSS-function ( $r=0.002, p=0.968$ and $r=0.014, p=0.823$, respectively) for either 
the first or second TKA. Likewise, the age at the time of the first TKA did not affect KSS-function $(r=-$ $0.081, p=0.190$ and $r=0.040, p=0.518)$ or WOMAC-physical function $(r=0.080, p=0.198$ and $r=0.041$, $p=0.509)$ in either knee.

\section{Discussion}

As a standard, successful surgery, BTKA accounts for a considerable proportion of all TKA performed to alleviate pain and improve physical function in patients with bilateral knee OA. In such patients, BTKA can be performed either simultaneously or as a staged procedure with a variable interval between surgeries. Compared with staged BTKA, simultaneous BTKA is associated with greater blood loss and a higher risk of medical complications and mortality [5]. However, there is no clearly established optimal time frame for performing the second surgery in staged BTKA, and this issue has not garnered much research attention.

One of the concerns regarding a short interval between staged surgeries is the possible increased risk of complications at the time of the second TKA, especially in patients with pre-existing cardiopulmonary disease or advanced age. Our first finding was that early complications of the second TKA did not differ significantly among time intervals. Our results were similar to previously reported findings. Yeh et al. found a non-significant trend toward higher complication and 90-day readmission rates when the second TKA was performed after 31-90 days [9]. Chen et al. could not identify a safe time frame for performing the second TKA, as the frequencies of complications among various time intervals did not differ significantly [10]. However, those two studies enrolled only patients who had the second TKA within 365 days after the first, even though $36 \%$ of patients with bilateral knee OA have contralateral surgery following unilateral TKA after a longer interval [16]. Ishii et al. reported that the median interval between the first and second staged BTKA operations was 12.5 months [17]. Crawford et al. evaluated shorter times between surgeries of 3-6,7-12,13-24, and $>24$ weeks in 1,005 patients who underwent staged BTKA [11]. They concluded that the time interval did not affect early medical or surgical complications after the second TKA, and that it is safe to proceed to the second stage at any time as long as the patients are medically stable. Notably, when comparing simultaneous and staged BTKA, Ritter et al. found that complications did not differ among time intervals, although staged bilateral BTKA at 3-6 months had the lowest mortality rate and fewest disadvantages [18].

Our second finding was that the long-term functions of the first and second knees were not significantly different for any time interval. Many studies of staged BTKA have shown that patients have inferior postoperative functional scores for the second-operated knee compared with the first in the short term [19-21]. Poultsides et al. [22] reported that patients' preoperative expectations increase for the second surgery compared with the first. This might be one of the main reasons for the poorer clinical outcomes of the second knee. Conversely, Qutob et al. reported that patients with bilateral knee OA commonly elect to have the most symptomatic knee done first [19]. Different symptoms between the two knees lead to different degrees of postoperative improvement, which in turn subjectively influences patient functional scores. In a meta-analysis comparing the clinical outcomes of staged BTKAs, Malahias et al. posited that 
any postoperative differences between the first and second knees would disappear with longer follow-up. Our results, and those of Lizaur-Utrilla et al. [7], confirm that hypothesis. Similar to our study, Lizaur-Utrilla et al. showed that BTKAs performed at different intervals yielded equivalent function scores between the knees at the 5-year follow-up, although the "mental score" and patient satisfaction were better for the second TKA.

Age and TKA type (PS vs. MP) did not influence our outcomes. A study comparing simultaneous and staged BTKA reported that age did not affect the postoperative Oxford Knee Score in either knee [23]. Several studies reported no significant differences in clinical results between the two prosthesis types among patients undergoing unilateral TKA $[24,25]$. No study has analysed whether these two different prostheses influence outcomes in patients with staged BTKA at different intervals. Lizaur-Utrilla et al. found no significant differences in KSS or WOMAC scores between cruciate-retaining and PS TKA [7].

Our study had several limitations that should be considered. Firstly, the study was retrospective; the complications of patients admitted to other medical institutions may not have been captured. Secondly, we included a relatively small sample, as the patients were recruited from a single hospital. Therefore, the study may have been underpowered. Hence, it will be necessary to conduct a multicentre, prospective, cohort trial to generate higher-level evidence to confirm our findings. In addition to staged and simultaneous BTKA, BTKA also can be staggered by a few days during a single hospitalisation. A recent systematic review reported that the use of staggered BTKA is continuing to decline, as it does not appear to confer a clinical advantage over simultaneous procedures [26]. Despite the lower total cost of the staggered BTKA method compared to staged BTKA, more research needs to explore whether it is safer and confers clinical advantages.

\section{Conclusion}

This study found no differences in early complications of the second TKA according to whether staged BTKA was performed after a short or long interval. Postoperative knee function was also equivalent and unaffected by the length of the surgical interval. Our results can help surgeons discuss the timing of the second TKA with patients, which should be based on their own preferences. If patients cannot tolerate severe symptoms in the contralateral knee after the first TKA, the second TKA should be performed as early as possible.

\section{Abbreviations}

BTKA, bilateral total knee arthroplasty; OA, osteoarthritis; KSS, Knee Society Score; WOMAC, Western Ontario and McMaster Universities Arthritis Index; BMI, body mass index; $\mathrm{CCl}$, Charlson Comorbidity Index; PJI, periprosthetic joint infection

\section{Declarations}




\section{Ethics approval and consent to participate}

This study was approved by Medical Ethics Committee of Affiliated Hospital of Qingdao University. Informed consent was obtained from all individual participants included in the study. All procedures performed in studies involving human participants were in accordance with the ethical standards of the institutional research committee and with the 1964 Helsinki declaration and its later amendments or comparable ethical standards

\section{Consent for publication}

Written informed consent was obtained from the patient for publication of this case report and any accompanying images. A copy of the written consent is available for review by the Editor of this journal.

\section{Availability of data and materials}

The final dataset will be available from the corresponding author.

\section{Competing interests}

The authors declare that they have no competing interests.

\section{Funding}

The study is supported by grants from National Natural Science Foundation of China (82002349). These funding bodies had role in the collection and interpretation of data and in writing the manuscript.

\section{Authors' contributions}

GS: wrote the paper. SX, CG, and FJ: collected the data and participated in writing and revising the paper. SY and KL: performed all the analysis. HX: designed the whole study.

\section{Acknowledgements}

N/A.

\section{References}

1. Lützner J, Hübel U, Kirschner S, Günther KP, Krummenauer F: [Long-term results in total knee arthroplasty. A meta-analysis of revision rates and functional outcome]. Der Chirurg; Zeitschrift fur alle Gebiete der operativen Medizen 2011, 82(7):618-624.

2. Memtsoudis SG, Ma Y, Chiu YL, Poultsides L, Gonzalez Della Valle A, Mazumdar M: Bilateral total knee arthroplasty: risk factors for major morbidity and mortality. Anesthesia and analgesia 2011, 113(4):784-790. 
3. Fu D, Li G, Chen K, Zeng H, Zhang X, Cai Z: Comparison of clinical outcome between simultaneousbilateral and staged-bilateral total knee arthroplasty: a systematic review of retrospective studies. The Journal of arthroplasty 2013, 28(7):1141-1147.

4. Tsay EL, Grace TR, Vail T, Ward D: Bilateral Simultaneous vs Staged Total Knee Arthroplasty: Minimal Difference in Perioperative Risks. The Journal of arthroplasty 2019, 34(12):2944-2949.e2941.

5. Hu J, Liu Y, Lv Z, Li X, Qin X, Fan W: Mortality and morbidity associated with simultaneous bilateral or staged bilateral total knee arthroplasty: a meta-analysis. Archives of orthopaedic and trauma surgery 2011, 131(9):1291-1298.

6. Bohm ER, Molodianovitsh K, Dragan A, Zhu N, Webster G, Masri B, Schemitsch E, Dunbar M: Outcomes of unilateral and bilateral total knee arthroplasty in 238,373 patients. Acta orthopaedica 2016, 87 Suppl 1(Suppl 1):24-30.

7. Lizaur-Utrilla A, Serna-Berna R, Vizcaya-Moreno MF, Martinez-Mendez D, Marco-Gomez L, Lopez-Prats FA: Comparison of Functional Outcomes Between the First and Second Knee in Staged Bilateral Total Knee Arthroplasty With Diverse Intervals Between Stages. The Journal of arthroplasty 2018, 33(9):2863-2867.

8. Yilmaz B, Sirin E, Kilinc BE, Ozdemir G, Komur B, Heybeli N: Investigation of the Optimal Interval for Staged Total Knee Arthroplasty for the Treatment of Advanced Bilateral Gonarthrosis. Acta chirurgiae orthopaedicae et traumatologiae Cechoslovaca 2020, 87(3):203-209.

9. Yeh JZY, Chen JY, Lee WC, Chong HC, Pang HN, Tay DKJ, Chia SL, Lo NN, Yeo SJ: Identifying an Ideal Time Frame for Staged Bilateral Total Knee Arthroplasty to Maximize Functional Outcome. The journal of knee surgery 2017, 30(7):682-686.

10. Chen AF, Rasouli MR, Vegari DN, Huang RC, Maltenfort MG, Parvizi J: Staged Bilateral Total Knee Arthroplasty: Time of the Second Side. The journal of knee surgery 2015, 28(4):311-314.

11. Crawford DA, Adams JB, Hurst JM, Morris MJ, Berend KR, Lombardi AV, Jr.: Interval Between Staged Bilateral Total Knee Arthroplasties Does Not Affect Early Medical or Surgical Complications. The Journal of arthroplasty 2021, 36(2):537-541.

12. Charlson ME, Pompei P, Ales KL, MacKenzie CR: A new method of classifying prognostic comorbidity in longitudinal studies: development and validation. Journal of chronic diseases 1987, 40(5):373383.

13. Parvizi J, Gehrke T: Definition of periprosthetic joint infection. The Journal of arthroplasty 2014, 29(7):1331.

14. Insall JN, Dorr LD, Scott RD, Scott WN: Rationale of the Knee Society clinical rating system. Clinical orthopaedics and related research 1989(248):13-14.

15. Whitehouse SL, Lingard EA, Katz JN, Learmonth ID: Development and testing of a reduced WOMAC function scale. The Journal of bone and joint surgery British volume 2003, 85(5):706-711.

16. Walmsley P, Murray A, Brenkel IJ: The practice of bilateral, simultaneous total knee replacement in Scotland over the last decade. Data from the Scottish Arthroplasty Project. The Knee 2006, 13(2):102-105. 
17. Ishii Y, Noguchi H, Takeda M, Sato J, Toyabe S: Time between the first and second operations for staged total knee arthroplasties when the interval is determined by the patient. The Knee 2014, 21(1):221-223.

18. Ritter M, Mamlin LA, Melfi CA, Katz BP, Freund DA, Arthur DS: Outcome implications for the timing of bilateral total knee arthroplasties. Clinical orthopaedics and related research 1997(345):99-105.

19. Qutob M, Winemaker M, Petruccelli D, de Beer J: Staged bilateral total knee arthroplasty: does history dictate the future? The Journal of arthroplasty 2013, 28(7):1148-1151.

20. Kim MH, Nahm FS, Kim TK, Chang MJ, Do SH: Comparison of postoperative pain in the first and second knee in staged bilateral total knee arthroplasty: clinical evidence of enhanced pain sensitivity after surgical injury. Pain 2014, 155(1):22-27.

21. Scott CE, Murray RC, MacDonald DJ, Biant LC: Staged bilateral total knee replacement: changes in expectations and outcomes between the first and second operations. The bone \& joint journa/2014, 96-b(6):752-758.

22. Poultsides LA, Ghomrawi HM, Lyman S, Aharonoff GB, Mancuso CA, Sculco TP: Change in preoperative expectations in patients undergoing staged bilateral primary total knee or total hip arthroplasty. The Journal of arthroplasty 2012, 27(9):1609-1615.e1601.

23. Abram SG, Nicol F, Spencer SJ: Patient reported outcomes in three hundred and twenty eight bilateral total knee replacement cases (simultaneous versus staged arthroplasty) using the Oxford Knee Score. International orthopaedics 2016, 40(10):2055-2059.

24. Shi W, Jiang Y, Wang C, Zhang H, Wang Y, Li T: Comparative study on mid- and long-term clinical effects of medial pivot prosthesis and posterior-stabilized prosthesis after total knee arthroplasty. Journal of orthopaedic surgery and research 2020, 15(1):421.

25. Kulshrestha V, Sood M, Kanade S, Kumar S, Datta B, Mittal G: Early Outcomes of Medial Pivot Total Knee Arthroplasty Compared to Posterior-Stabilized Design: A Randomized Controlled Trial. Clinics in orthopedic surgery 2020, 12(2):178-186.

26. Malahias MA, Gu A, De Martino I, Selemon NA, Ast MP, Sculco PK: Staggered bilateral total knee arthroplasty during a single hospitalization: is it still an option? a systematic review. Musculoskeletal surgery 2021. 